\title{
Nontarget Metabolomics of Grape Seed Metabolites Produced by Various Scion-Rootstock Combinations
}

\author{
Zhijun Zhang, Huaifeng Liu, Junli Sun, Songlin Yu, Wang He, and Tianyuan Li \\ Department of Horticulture, College of Agriculture/The Key Laboratory of Characteristics of Fruit \\ and Vegetable Cultivation and Utilization of Germplasm Resources of the Xinjiang Production and \\ Construction Corps, Shihezi University, Shihezi 832003, People's Republic of China
}

\section{Zhao Baolong}

The Key Laboratory of Oasis Ecoagriculture, Xinjiang Production and Construction Corps, Shihezi University, Shihezi 832003, People's Republic of China

\begin{abstract}
ADDITIONAL INDEX wORDS. differential metabolites, liquid chromatography-mass spectrometry, Vitis vinifera
ABstract. The use of resistant rootstocks is an inevitable trend in the development and production of grapes (Vitis sp.). The present study analyzed differences in the metabolites in grape seeds of different rootstock combinations (1103P, 5C, SO4, 3309C, 140R, and control) grafted onto 'Cabernet Sauvignon' (CS) wine grape (Vitis vinifera) scions (control, CS/CS, self-rooted grafting vines) using liquid chromatography-mass spectrometry (LC-MS) and nontargeted metabolomic techniques. Principal component analysis (PCA), partial least squares discriminant analysis (PLS-DA), and orthogonal-partial least squares discriminant analysis identified 30 significant metabolites and 22 metabolic pathways in the seeds of CS that differed significantly from the control seeds. This study revealed that rootstocks influence metabolite concentrations and metabolic pathways (alanine-aspartate-glutamate pathway, arginine-proline pathway, and the tricarboxylic acid cycle) in the scion onto which they are grafted. The rootstocks increased the concentration of delphinidin-3-(6-acetylglucoside), peonidin 3-(6-p-coumarylglucoside), L-threonine, and D-tartaric in CS seeds. Appropriate rootstock combinations can be used to improve the quality of grape seeds by changing the concentrations of amino acids, organic acids, polyphenols, and vitamin $B$. This study provides a theoretical basis for selecting grape rootstocks and provides important insights for improving the quality of commercial products derived from grape seeds.
\end{abstract}

Grapes (Vitis sp.) are widely cultivated for fresh or dried fruit, wine making, juice making, and as a components of health foods and medicines. In fact, grapes are one of the most commercially valuable plants worldwide (Harb et al., 2015; Leng et al., 2015). The total land area used for grape cultivation has been increasing over the past 10 years in China, and China is now the leading grape producer worldwide (International Organisation of Vine and Wine, 2018). The Xinjiang region, historically China's largest grape-producing region, covers a vast territory with a diverse array of climates and soils. The region's long history (2400 years) of producing grapes and wines is attributed to its climatic conditions being suitable for grape production. Drought, soil salinity, and cold weather can severely impact the development and production of wine grape $(V$. vinifera), and therefore, a cultivar of rootstocks has been developed in Xinjiang grape to resist such environmental stress. These resistant rootstocks not only improve the stress resistance of scions but also affect the yield and quality of grape berries

Received for publication 15 Oct. 2019. Accepted for publication 19 Mar. 2020. Published online 28 April 2020.

This research was supported by the Natural Science Foundation of China (31560542).

We thank www.enago.com for editorial assistance with the English. We thank BioNovGene for technical assistance.

Z.Z. and Z.B. are co-first authors.

J.S. and S.Y. are the corresponding authors.

E-mail: 1530322722@qq.com or songlin8900@sina.com.

This is an open access article distributed under the CC BY-NC-ND license (https://creativecommons.org/licenses/by-nc-nd/4.0/).
(Corso et al., 2016; Suriano et al., 2016). Rootstock and scion combinations can also be used to regulate vine growth, phenological period, fruit yield, and the concentrations of key constituents in the fruit, such as tannins, anthocyanins, and resveratrol, all of which affect grape quality and taste by changing water balance relationships, gas exchange rates, and nutrient absorption capacities. Areas under grape cultivation can be increased in China (and the commercial industry enhanced) if grape cultivars are improved to enable growth under varied soil and climatic conditions, further resist pathogens, and improve the concentrations of key chemical constituents.

Grape seeds comprise flavonoids and other metabolites that improve plant biotic and abiotic resistance. They also have bactericidal and antiviral effects and potential roles in preventing cardiovascular and cerebrovascular diseases (Zerbib et al., 2018). Trad et al. (2017) think polyphenols in grape seeds are key compounds that play a role in selection and domestication, as they offer astringency and bitterness to derived products and promote health safety. Tannins, monomeric flavan-3-ols and other substances in the seed are correlated to wine quality by both consumer and expert tasting panels, which have an effect on the color and taste of wine (Joshua et al., 2020). At the same time, grape seed is the primary by-product of wine production. Because of their high content of phenols, unsaturated acids, and other biologically active compounds, grape seeds have been increasingly used for further processing; for instance, they are processed into high-quality plant oil. It is also believed that 
grape seeds have a health function and an effect of extending the shelf life of food (Gordana et al., 2019; Hela et al., 2018). Approximately 155 cosmetics have "grape seed" in their names in the China Food and Drug Administration database, and 127 types of health foods have been named "grape seed." Grape seeds have become an important source of chemicals for some foods, health products, and medicines. At present, research on the effects of rootstocks on scion quality is mainly focused on the growth and development of vines and only partially on fruit quality. However, no studies have been conducted on the effects of various rootstocks on the chemical composition of grape seeds or related metabolic processes and pathways. Metabolomic profiling involves the analysis and data integration of a total population of metabolites in a given sample based on high-throughput, multivariate data. Nontarget metabolomics analyzes the broadest possible array of metabolites in a sample from a holistic perspective and has been routinely used in science, including in biology, clinical medicine, pharmacology, and food science (Arapitsas et al., 2016; Narduzzi et al., 2015; Qi et al., 2011). When Bueno et al. (2018) used metabolomic profiling to evaluate organically and conventionally cultivated tomato (Solanum lycopersicum), they found that organically cultivated tomato exhibited significantly different chemical sample profiles for six bioactive compounds: L-tyrosyl-L-isoleucyl-L-threonyl-L-threonine, trilobatin, phloridzin, tomatine, phloretin, and echinenone. Harb et al. (2015) used gas chromatography-mass spectrometry and LCMS to examine the effects of abiotic stress on the metabolism of various grape cultivars and found that stress significantly affected primary metabolite production (especially amino acids) but had no effects on secondary metabolite production. When Son et al. (2008) found significant differences in the chemical compositions of several grape cultivars grown in various grape-producing areas using metabolomics, they suggested that metabolomics could be used to predict wine quality and product origin. Other studies have also demonstrated that metabolomic profiling can be used to effectively analyze variations in grape metabolites and identify differences in metabolic pathways among grape cultivars.

In this study, nontarget LC-MS was used to study the effect of rootstock grafting on the grape seed metabolome of CS, a major wine grape cultivar in China. This study used PCA and partial multivariate statistical analysis methods [e.g., PLS-DA] to screen differences in grape metabolites among various rootstock combinations and analyze their metabolic pathways, and explored patterns in metabolite accumulation to analyze grape seed quality and the nutritional effects of various grape rootstocks on CS seeds. By evaluating the effects of scionrootstock graft combinations, this study will provide a theoretical basis and provide important insights for improving the quality of commercial grape seed-related products.

\section{Materials and Methods}

Test materials. This study used CS as scion material. A cultivar of rootstocks was grafted onto $\mathrm{CS}$, which provides excellent resistance: rootstocks 'SO4' and '5C' (Vitis berlandieri $\times$ Vitis riparia), rootstocks '140R' and '1103P' (V. berlandieri $\times V$. rupestris), and rootstock '3309C' ( $V$. riparia $\times V$. rupestris). The rootstock combinations used in our experiments were $\mathrm{CS} /{ }^{\prime} 1103 \mathrm{P}$ ', $\mathrm{CS} /{ }^{\prime S O} 4$ ', $\mathrm{CS} /{ }^{\circ} 140 \mathrm{R}$ ', $\mathrm{CS} /{ }^{\prime} 5 \mathrm{C}$ ', and $\mathrm{CS} /{ }^{\prime} 3309 \mathrm{C}$ ', with $\mathrm{CS} / \mathrm{CS}$ (self-rooted grafting seedlings) used as the control. All rootstocks were obtained from the National Grape Resources of Zhengzhou Fruit Research Institute.

In Autumn 2015, 10 hard-twig vines with uniform growth were selected for each rootstock scion combination; these were grafted and planted $(0.5 \mathrm{~m}$ apart $)$ in blocks in the field at the Experimental Station of the Agricultural College of Shihezi University in Xinjiang, China (lat. $86^{\circ} 06^{\prime} \mathrm{N}$, long. $44^{\circ} 32^{\prime} \mathrm{E}$ ). Cultivation conditions and management were consistently maintained. The cultivation management conditions were the same, and the control group has similar processing yields. In Sept. 2017, fully ripe grape bunches were randomly selected from near the root and away from the root, with $\approx 400 \mathrm{~g}$ of berries per replicate and five replicates per each rootstock scion combination. Seeds were quickly collected from the fruit in the laboratory by hand, packaged in tin foil, frozen in liquid nitrogen, and stored at $-80^{\circ} \mathrm{C}$. This study used ultrapure water [ $\mathrm{ddH}_{2} \mathrm{O}$ (Arium mini; Sartorius, Göttingen, Germany)] and chromatographically pure methanol, chloroform (Sinopharm Chemical Reagent Co., Shanghai, China), acetonitrile (Merck Chemical Technology Shanghai Co., Shanghai, China), and formic acid (Tokyo Chemical Industry Co., Tokyo, Japan) as reagents.

INSTRUMENTS AND EQUIPMENT. The experimental instruments included a vacuum concentrator (53050; Eppendorf, Hamburg, Germany), a centrifuge (H1650-W; Eppendorf), a vortex mixer (QL-866; Qilinbeier, Haimen, China), a pure water meter (Arium mini), a $0.22-\mu \mathrm{m}$ polytetrafluoroethylene filter (Jin Teng, Tianjing, China), an ultrasonic cleaner (KW-100TDV; Shumei, Kunshan, China), and a tissue grinder (SCIENTZ-48; Xinzhi, Ningbo, China).

SAMPle PREPARATION. For each treatment, $0.5 \mathrm{~g}$ of grape seed was ground using a high-flux tissue grinder, $100 \mathrm{mg}$ of grape seed powder was placed into a 5-mL centrifuge tube, and then $1000 \mu \mathrm{L}$ of methanol $\left(-20{ }^{\circ} \mathrm{C}\right)$ was added; this mixture was vortexed for $30 \mathrm{~s}$ and the vortexed material was placed in an ultrasonic cleaner for $30 \mathrm{~min}$ at room temperature. Then, 750 $\mu \mathrm{L}$ chloroform and $800 \mu \mathrm{L} \mathrm{ddH}_{2} \mathrm{O}$ (at $4{ }^{\circ} \mathrm{C}$ ) were added to the mixture, which was vortexed for $1 \mathrm{~min}$ and centrifuged for 10 min at $12,000 g_{\mathrm{n}}$ and $4{ }^{\circ} \mathrm{C}$. A total of $1000 \mu \mathrm{L}$ of supernatant was removed and transferred to a new $1.5-\mathrm{mL}$ centrifuge tube, and it was concentrated using a vacuum centrifugation concentrator. The sample was dissolved in $250 \mu \mathrm{L}$ methanol $/ \mathrm{ddH}_{2} \mathrm{O}$ solution $\left[1: 1(\mathrm{v} / \mathrm{v})\right.$ at $\left.4{ }^{\circ} \mathrm{C}\right]$ and filtered through a $0.22-\mu \mathrm{m}$ membrane filter. Subsequently, $20 \mu \mathrm{L}$ of each sample was removed and mixed with a quality control sample to correct for any deviations in the mixed samples due to error(s) caused by the analytical instrument. LC-MS was performed on the remaining samples tested. Five replicates were then randomly selected for each treatment (De Vos et al., 2007).

Chromatography conditions. This study used an ultraperformance liquid chromatography system [UPLC (Acquity; Waters, Milford, MA)] equipped with a $1.8-\mu \mathrm{m}, 150 \times 2.1-\mathrm{mm}$ column (Acquity UPLC HSS T3; Waters) maintained at $40{ }^{\circ} \mathrm{C}$ to chromatographically separate metabolites. The temperature of the autosampler was maintained at $4{ }^{\circ} \mathrm{C}$. The gradient elution of analytes was performed using $0.1 \%$ formic acid in water (Solvent A) and $0.1 \%$ formic acid in acetonitrile (Solvent B) at a flow rate of $0.25 \mathrm{~mL} \cdot \mathrm{min}^{-1}$. After equilibration, $6 \mu \mathrm{L}$ of each sample was injected. An increasingly linear gradient of Solvent $\mathrm{B}(\mathrm{v} / \mathrm{v})$ was used as follows: $2 \%$ Solvent B for the first minute, from $2 \%$ to $50 \%$ Solvent B from min 1 to $\min 9.5$, from $50 \%$ to $98 \%$ Solvent B from min 9.5 to min 14, 98\% Solvent B from 
$\min 14$ to $\min 15$, from $98 \%$ to $2 \%$ Solvent B from min 15 to min 15.5 , and $2 \%$ Solvent B from min 15.5 to min 17 (Sangster et al., 2006).

Mass Spectrometry Conditions. This study used electrospray ionization mass spectrometry $\left(E S I-M^{n}\right)$ using a mass spectrometer (LTQ-Orbitrap XL; Thermo Fisher Scientific, Waltham, MA) with positive and negative spray voltages at 4.8 and $-4.5 \mathrm{kV}$, respectively. Sheath gas and auxiliary gases were set at 45 and 15 arbitrary units, respectively. The capillary temperature was $325^{\circ} \mathrm{C}$. The positive and negative voltages of the capillary were set at 35 and $-15 \mathrm{~V}$, respectively, whereas those of the tube were set at 50 and $-50 \mathrm{~V}$, respectively. We used an Orbitrap analyzer (Thermo Fisher Scientific) scanned over a mass range of 50 to $1000 \mathrm{~m} / \mathrm{z}$ for a full scan. We performed data-dependent acquisition MS/MS experiments with a collision-induced dissociation scan. The normalized collision energy was $30 \mathrm{eV}$. Dynamic exclusion was implemented with a repeat count of 2 and exclusion duration of $15 \mathrm{~s}$ (Want et al., 2010).

DAta ANALYsis. Data were analyzed using proteomics data analysis software (ProteoWizard version 3.0; ProteoWizard, Palo Alto, CA), and mass spectrometric peaks were filtered, identified, and aligned using statistical analysis software ( $R$ version 3.3; R Foundation for Statistical Computing, Vienna, Austria) to obtain data matrices for mass-to-core ratios, retention times, and peak areas. The data were then subjected to batch normalization and adaptive conversion to standardize the peak areas. PCA [SIMCA-P (version 13.0; Umetrics AB, Umea, Sweden) and R language ropes package], PLS-DA, orthogonal PLS-DA (OPLS-DA), and other chemometric principles and multivariate statistical analyses were performed (Thévenot et al., 2015; Want et al., 2010).

\section{Results and Discussion}

\section{Differences in the metabolome of CS grape seeds of various rootstock combinations}

PCA AnAlysis. In the present study, the PCA scores reflect the original state of the metabolome data, which was helpful in examining the differences in metabolites produced in grape seeds of various CS scion-rootstock combinations and all the data at once (Fig. 1) (Gromski et al., 2015). There was a wide separation of grape seed samples from the grafts of CS/ '3309C', CS/'1103P', CS/'SO4', and CS/CS (control) on the ordination diagram, indicating obvious differences in metabolite production among the various rootstock grafts. The $\mathrm{CS} /$ ' $140 \mathrm{R}$ ' and $\mathrm{CS} /{ }^{\prime} 5 \mathrm{C}$ ' rootstock combinations did not separate, indicating that there is no significant difference between their metabolites.

PLS-DA ANALYsis. PCA analysis considers intragroup errors and does not eliminate random errors unrelated to the research purpose, indicating that PCA is not very conducive for detecting differences between groups. Therefore, this study used a PLS-DA to further classify the grape seed metabolites derived from the rootstock combinations (Boulesteix and Strimmer, 2007; Lee et al., 2010).

The PLS-DA score plot and permutation test results of various rootstock combinations of CS grape seed samples was essentially consistent with our PCA results (Fig. 2). The principal component regression coefficient (Q2) for grape seed samples was 0.962 , which is much more than 0.5 (maximum value $=1.0)$, indicating that the model is extremely predictive,

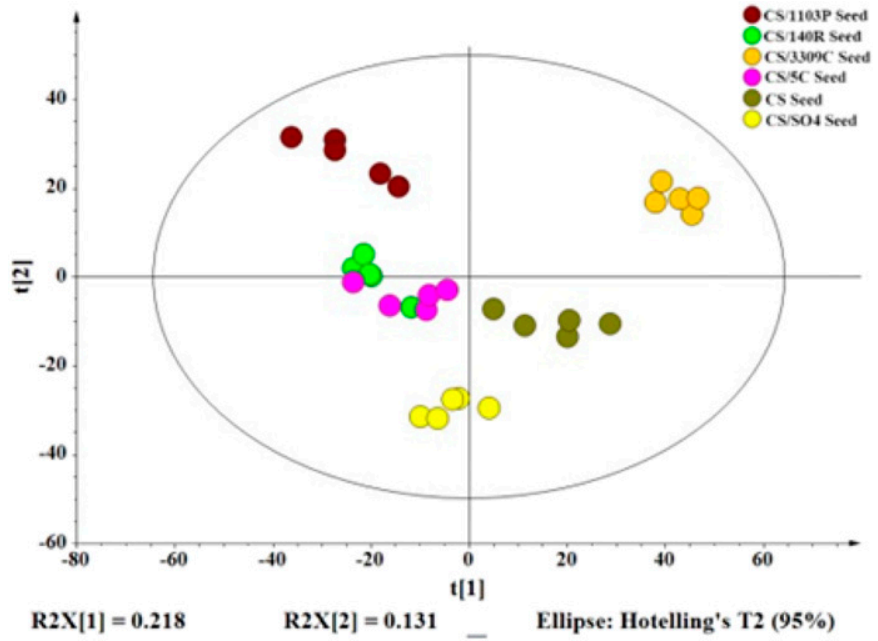

Fig. 1. Principal components analysis (PCA) scores of 'Cabernet Sauvignon' (CS) grape seeds of various rootstock combinations.

reflecting the visually obvious geometric separation between samples (i.e., rootstock combinations) in the PCA diagram. The percent contribution (R2Y) of the model of CS grape seed samples was 0.992 , indicating that the contribution of the model to the variation in the dependent variables was $99.2 \%$. When combining data from various rootstocks with a CS grape seed model (based on 100 permutation test experiments), the Q2 point of the model was moved lower than the original Q2 point (from the left to the right) and the R2 and Q2 values on the rightmost side were both more than 0.9 . Furthermore, the intercept of the Q2 regression line was -0.594 , which indicates that the constructed PLS-DA model is not associated with overfitting, and that the model has good predictive ability, is effectively available, and can combine CS with different rootstocks.

OPLS-DA ANALYSIS. OPLS-DA, which is an extension of PLS-DA, is an analytical method that is commonly used when analyzing metabolomics data (Consonni et al., 2009; Tarachiwin et al., 2008). The plots of OPLS-DA scores (Fig. 3) were generally consistent with the plots of PCA scores. The OPLSDA model explained $62.5 \%$ of the variation in the independent variable $\mathrm{X}(\mathrm{R} 2 \mathrm{X}=0.625)$ and $99.2 \%$ of the variation in the categorical variable $\mathrm{Y}(\mathrm{R} 2 \mathrm{Y}=0.992)$, with a $\mathrm{Q} 2$ value of 0.932 , indicating that the model explained $93.2 \%$ of sample variation. These results demonstrate that the OPLS-DA model is stable and has strong predictive power. Although data from the CS/ ' $140 \mathrm{R}$ ' and CS/'5C' seeds have partial overlap in PCA or PLSDA and no overlap in the OPLS-DA score plot, the separation effect is better. All the samples separated from one another in OPLS-DA, suggesting differences in the metabolites produced in seeds owing to the differences in the various rootstocks onto which the CS scions were grafted.

\section{Screening and identification of metabolites}

The variable importance in projection values of variables greater than 1.0 (VIP $>1$ ) and one-way analysis of variance were used to identify significant differences $(P \leq 0.05)$ in grape seed metabolites among the various rootstocks of CS. Six of the 30 metabolites (Table 1) discovered in grape seeds significantly differed among the various rootstock combinations evaluated (Fig. 4). 

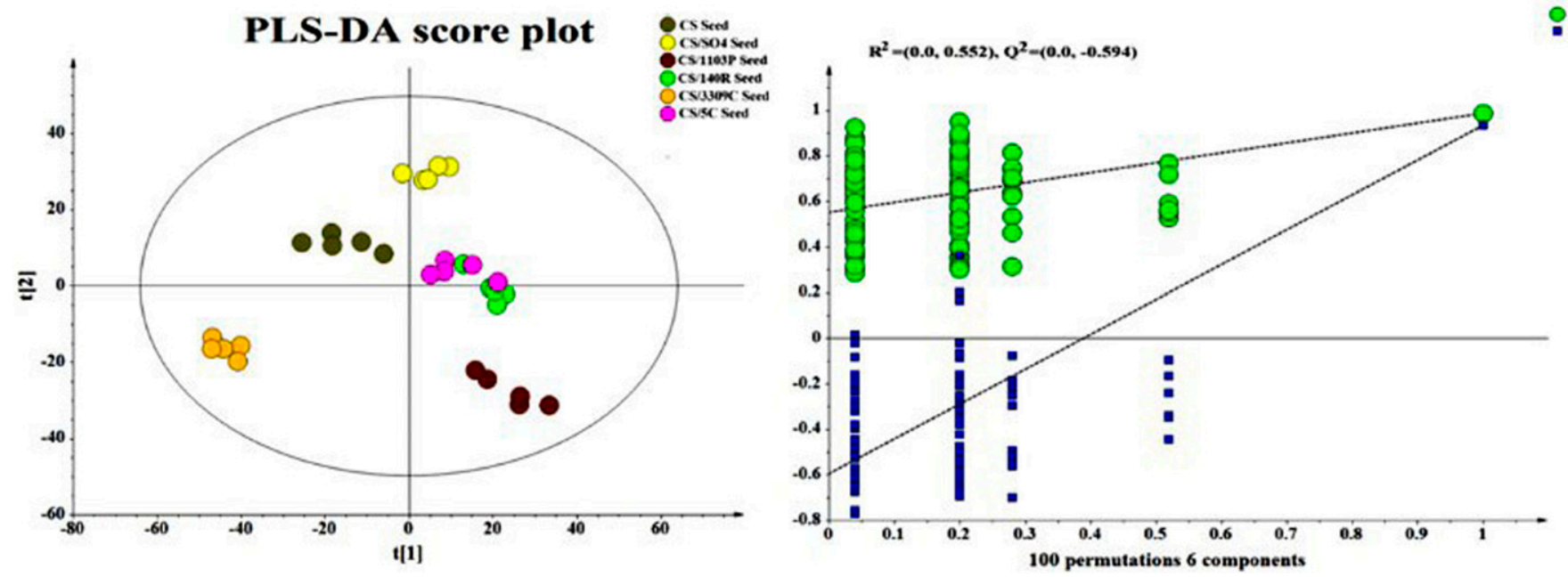

Fig. 2. Partial least squares discrimination analysis (PLS-DA) score plot and permutation test results of 'Cabernet Sauvignon' (CS) grape seeds from various rootstock combinations.

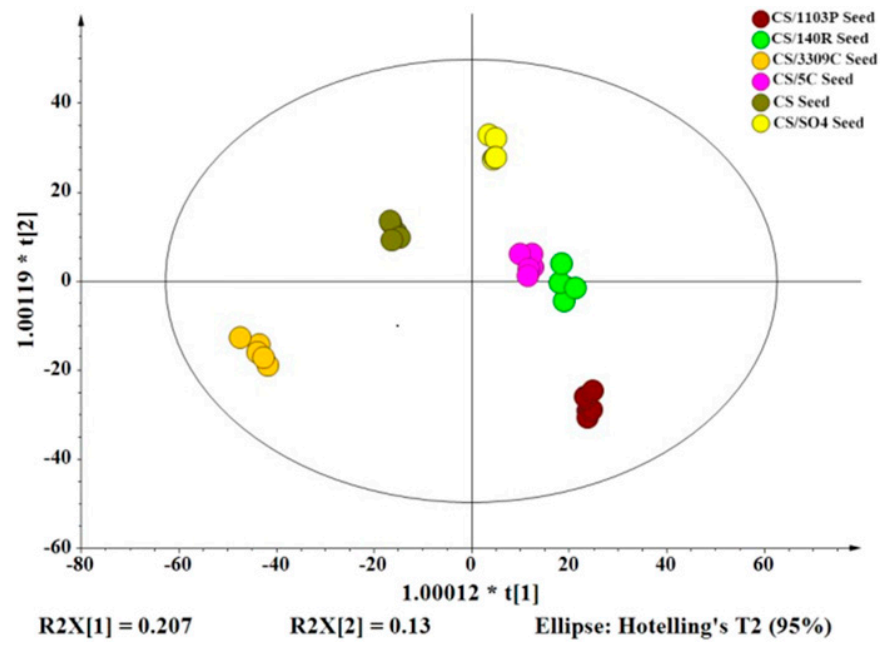

Fig. 3. Orthogonal-partial least squares discrimination analysis (OPLS-DA) score plot of 'Cabernet Sauvignon' (CS) grape seeds from various rootstock combinations.

\section{Heat map metabolites}

The cluster and heat map analyses of the metabolites for various rootstock combinations of CS grape seeds are shown in Fig. 5. The colors in the figure indicate relative metabolite concentration, with red indicating high concentration and green indicating low concentration. Each rootstock combination shows either significantly high or low areas of expression. The different rootstock combinations can be distinguished based on the relative expression of the above differential metabolite in grape seeds.

The concentration of malic acid was higher in the self-rooted vine (control group) than in other rootstock combinations (Fig. 4). Similarly, the concentration of alvidin-3-O-arabinoside was higher in the CS/'1103P' graft than in other rootstock combinations, and the concentrations of L-lysine, malvidin 3-(6-pcaffeyglucoside), kaempferol 3-O-beta-D-glucoside, gallate, citric acid, and 5-oxo-D-proline were higher in the CS/'SO4' combination than in other rootstock combinations. The con- centrations of malvidin 3-(6-p-coumarylglucoside), N-acetylL-glutamate, L-arginine, pyridoxine, and adenosine were higher in the $\mathrm{CS} / 140 \mathrm{R}$ rootstock combination than in other combinations, and the concentrations of L-glutamic acid, (-)-epicatechin, L-threonine, myricetin 3-glucoside, and petunidin 3-(6-acetylglucoside) were higher in the $\mathrm{CS} /$ ' $5 \mathrm{C}$ ' combination than in other combinations. The concentrations of 2-oxoglutaric acid, D-gluconic acid, (R)-pantothenic acid, 1-(3-hydroxy-4-5dimethoxybenzoyl) pyranose, peonidin 3-(6-p-coumarylglucoside), 2-deoxy-alpha-D-ribopyranose, and delphinidin 3-(6-acetylglucoside) were higher in the $\mathrm{CS} /{ }^{\prime} 3309 \mathrm{C}$ ' combination than in other combinations.

\section{Pathway analysis of metabolic products}

This study analyzed the metabolic pathways based on the variations in the metabolites in grape seeds within the various rootstock combinations examined. This study used the Metabolic Pathway Analysis (MetPA) (based on the Kyoto Encyclopedia of Genes and Genomes metabolic pathway database) to identify possible metabolic pathways affected by the various rootstock combinations (Kanehisa and Goto, 2000). This study found 15 substances enriched in metabolic pathways, including lysine, threonine, and glutamic acid, among which three amino acids (lysine, threonine, and glutamic acid) were involved in three or more different metabolic pathways (Table 2). This suggests that these three amino acids are positioned at nodes in metabolic pathways and so are the key substances connecting the various pathways. These three amino acids are precursors for the synthesis of various proteins/enzymes that are associated with tolerance to cold and resistance to closely related diseases.

The metabolic pathways altered by various rootstock combinations in grape seeds mainly involve 22 pathways associated with the metabolism of alanine, aspartic acid, and glutamate; the metabolism of arginine and proline; and the tricarboxylic acid (TCA) cycle (Fig. 6, Table 2). The three metabolic pathways with obvious combination effects (alanine, aspartic acid, and glutamic acid pathways) provide precursors for the synthesis of various amino acids that typically occur in grapes. These amino acids also constitute 
Table 1. Various metabolites of 'Cabernet Sauvignon' grape seeds of various scion-rootstock combinations.

\begin{tabular}{|c|c|c|c|c|c|}
\hline No. & Metabolite & $\mathrm{Mz}$ & Rt (s) & VIP & q-value \\
\hline 1 & D-gluconic acid & 195.0488 & 98.2249 & 1.12367 & 0 \\
\hline 2 & 2-oxoglutaric acid & 145.0128 & 156.2850 & 1.04388 & 0 \\
\hline 3 & N-acetyl-L-glutamate(2-) & 188.0542 & 211.8410 & 1.35998 & 0 \\
\hline 4 & 5-oxo-D-proline & 128.0344 & 189.0410 & 1.44629 & 0 \\
\hline 5 & Peonidin 3-(6-acetylglucoside) & 505.1314 & 426.2520 & 1.18307 & 0 \\
\hline 6 & L-arginine & 175.1183 & 95.4428 & 1.27279 & 0 \\
\hline 7 & (-)-epicatechin & 289.0676 & 372.4270 & 1.55669 & 0 \\
\hline 8 & Gallate & 169.0125 & 271.1590 & 1.20819 & 0 \\
\hline 9 & citric acid & 191.0174 & 172.4240 & 1.34724 & 0 \\
\hline 10 & nicotinic acid & 124.0387 & 158.7510 & 1.15311 & 0 \\
\hline 11 & L-lysine & 147.1120 & 84.1111 & 1.44138 & 0 \\
\hline 12 & L-threonine & 120.0649 & 101.5455 & 1.27202 & 0 \\
\hline 13 & 2-deoxy-alpha-D-ribopyranose & 133.0496 & 246.4400 & 1.23969 & 0 \\
\hline 14 & Petunidin 3-(6-acetylglucoside) & 521.1257 & 405.0090 & 1.25473 & 0 \\
\hline 15 & Delphinidin 3-(6-acetylglucoside) & 507.1101 & 382.6990 & 1.33976 & 0 \\
\hline 16 & (R)-pantothenic acid & 218.1005 & 304.4305 & 1.23284 & 0 \\
\hline 17 & kaempferol 3-O-beta-D-glucoside & 447.0877 & 476.3015 & 1.40111 & 0 \\
\hline 18 & pyridoxine & 170.0803 & 189.9590 & 1.2264 & 0 \\
\hline 19 & 1-O-(3-Hydroxy-4,5-dimethoxybenzoyl)hexopyranose & 359.0936 & 318.2920 & 1.02898 & 0 \\
\hline 20 & malic acid & 133.0131 & 115.0870 & 1.17395 & 0 \\
\hline 21 & Sequiterpene Lactone 326 & 265.1421 & 344.8430 & 1.10369 & 0 \\
\hline 22 & D-tartaric acid & 149.0074 & 105.8605 & 1.1301 & 0 \\
\hline 23 & L-glutamic acid & 148.0596 & 101.8770 & 1.18473 & 0 \\
\hline 24 & Myricetin 3-glucoside & 481.0951 & 418.1780 & 1.10691 & 0.00001 \\
\hline 25 & adenosine & 268.1026 & 248.3980 & 1.09745 & 0.00002 \\
\hline 26 & malvidin-3-O-arabinoside & 463.1209 & 400.6830 & 1.10058 & 0.00008 \\
\hline 27 & taurine & 125.9856 & 84.0558 & 1.23672 & 0.00028 \\
\hline 28 & Malvidin 3-(6-p-caffeyglucoside) & 655.1618 & 445.4310 & 1.05048 & 0.00475 \\
\hline 29 & Peonidin 3-(6-p-coumarylglucoside) & 609.1569 & 472.9930 & 1.01011 & 0.00797 \\
\hline 30 & Malvidin 3-(6-p-coumarylglucoside) & 639.1667 & 472.9930 & 1.00033 & 0.0121 \\
\hline
\end{tabular}

$\mathrm{Mz}=$ mass-to-charge ratio, $\mathrm{Rt}=$ retention time, $\mathrm{VIP}=$ variable importance of the first principal component of orthogonal-partial least squares discriminant analysis, q-value $=$ significant difference $P$ value after false-positive correction.

the main trait examined by commercial growers to predict growth characteristics in grapes. The arginine and proline metabolic pathways include amino acids that produce important substances that promote plant growth and development for producing cold-resistant osmotic constituents; and the TCA cycle is a common pathway for the oxidative metabolism of sugars, lipids, and proteins, and its intermediate products are precursors for the biosynthesis of amino acids, proteins, and fatty acids. This study found that plant-related amino acid concentration significantly increased after grafting the CS scion onto rootstock. Altering the metabolic pathways of alanine, aspartic acid, and glutamate, those of arginine and proline metabolism, and the TCA cycle increased the synthesis and enrichment of nutrients in grape seeds.

\section{Analysis of metabolites in grape seed relative to various rootstock combinations}

Grapes are widely used in fresh foods, juice and wine making, and pharmaceutics. Grape metabolites are also important in influencing the quality of grape seed-derived products, while also affecting the growth and development of grapes. To ensure the quality and safety of products that contain grapes, it is necessary to improve our understanding of the nutritional value of metabolites present in grapes.
Amino acids play an important nutritional role in grape seeds, in the taste of grape fruit, and in the timing of phenophases in grapes. For example, lysine is highly correlated with sugar concentration (Jin et al., 2016a), glutamate has a pleasant taste, and the ratio of proline-toarginine affects the number of days required for grapes to ripen (Jogaiah et al., 2013). Arginine regulates the nitric oxide synthase pathways, which increases the resistance of fruit to diseases (Ji et al., 2018). Arginine and lysine are also purported to improve cardiovascular health, whereas threonine is an essential amino acid that appears to exhibit antibacterial effects in humans (Rieping and Hermann, 2006; Zhao et al., 2015). Some rootstocks have been found to change the amino acid concentration in fruit of 'Gold Finger' (Jin et al., 2016b), 'Summer Black' (Jin et al., 2016a), and 'Thompson Seedless' table grapes (Ji et al., 2018). This study found that the concentrations of arginine, lysine, threonine, and glutamic acid were increased or decreased in seeds in varying degrees depending on the rootstock combination from which they were extracted (Fig. 4); for example, the concentration of those four amino acids in the grape seeds varied in the $\mathrm{CS} /$ 'SO4' rootstock, but other rootstock combinations exhibited high amino acid concentrations. Therefore, different rootstock combinations can regulate the amino acid concentration in grape seeds.

Organic acids are closely associated with the quality of grapes. For wine grapes, organic acids play an important role in the flavor composition of wine. Low content of organic acids in wine leads to increased acidity and affects the wine taste balance and quality (Batista et al., 2010). Citric acid, tartaric acid, and malic acid are the main organic acids in grape seeds, accounting for more than $90 \%$ of their total organic acid concentrations. These acids play a major role in controlling the flavor and acidity of grape fruit, (e.g., determining the sensory perception of wine and its $\mathrm{pH}$ ) and are widely used in food products. Overall, the acidity of tartaric acid is $90 \%$ of citric acid, and the acidity of malic acid is $80 \%$ of citric acid. Thus citric acid has a greater effect on acidity (Jin et al., 2016b). In the present study, the concentration of malic acid was lower (in varying degrees) than that in the control (self-rooted grafting) in each grafting combination, whereas the concentration of tartaric acid was higher (in varying degrees) than that in the control (self-rooted grafting). The $\mathrm{CS} /{ }^{\circ} \mathrm{SO} 4$ ' rootstock combination had a very significantly higher $(P<0.01)$ concentration of citric acid than the $\mathrm{CS} / \mathrm{CS}$ combination, and the $\mathrm{CS} /$ '1103P', CS/'140R' and CS/'5C' rootstock combinations had a 

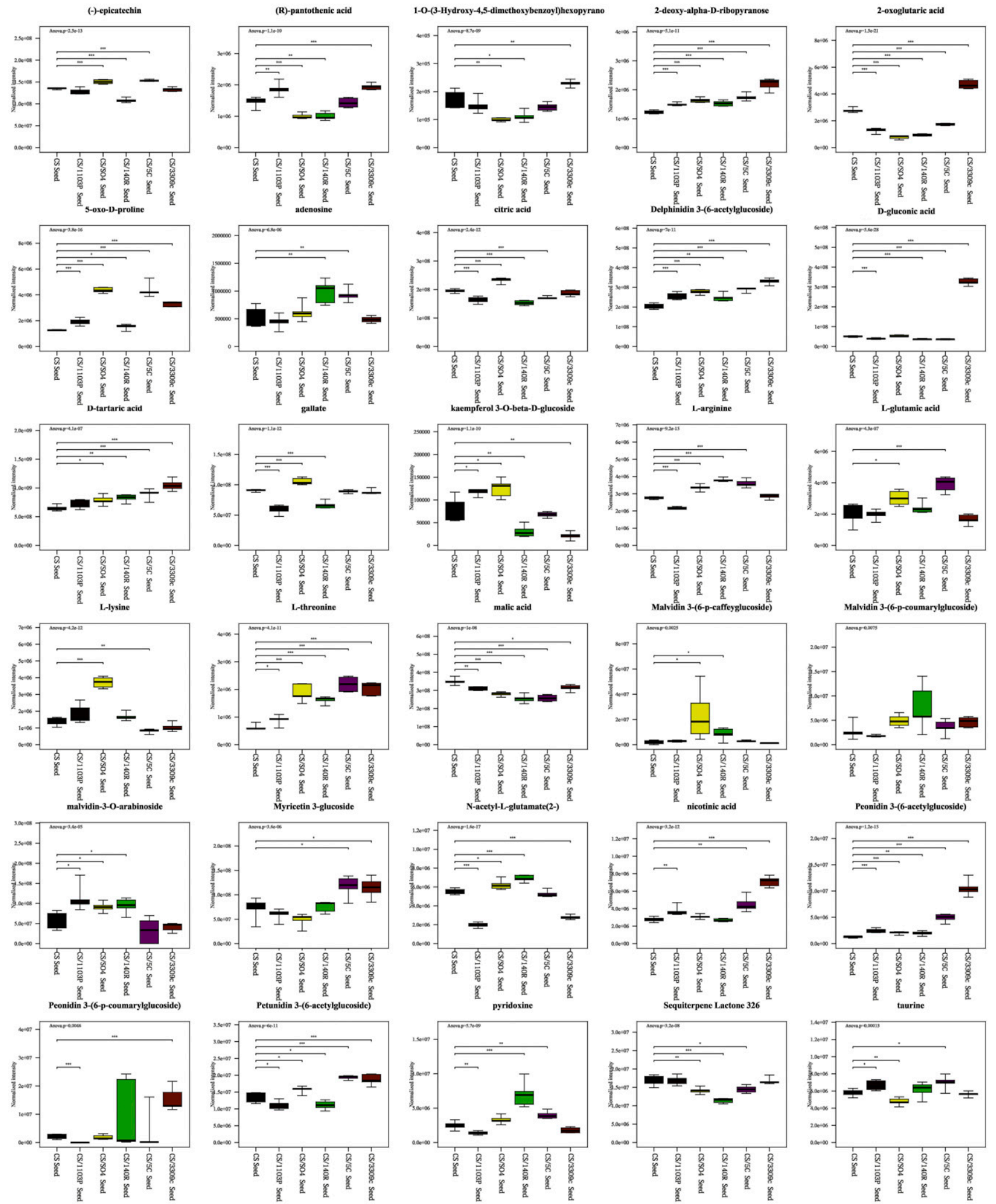

Fig. 4. Box diagram of differential metabolite concentrations in 'Cabernet Sauvignon' (CS) grape seeds from various rootstock combinations.

very significantly lower $(P<0.01)$ concentration of citric acid than the CS/CS combination. All the rootstock combinations, except the CS/'1103P' combination had significantly reduced concentrations of 2-oxoglutaric acid. It has been reported that the organic acid concentrations of the table grapes 'Gold Finger' (Jin et al., 2016a) and 'Summer Black' (Jin et al., 2016b), and the wine grape 'Sauvignon Blanc' (Pulko et al., 2012) were significantly affected by the different rootstocks, 


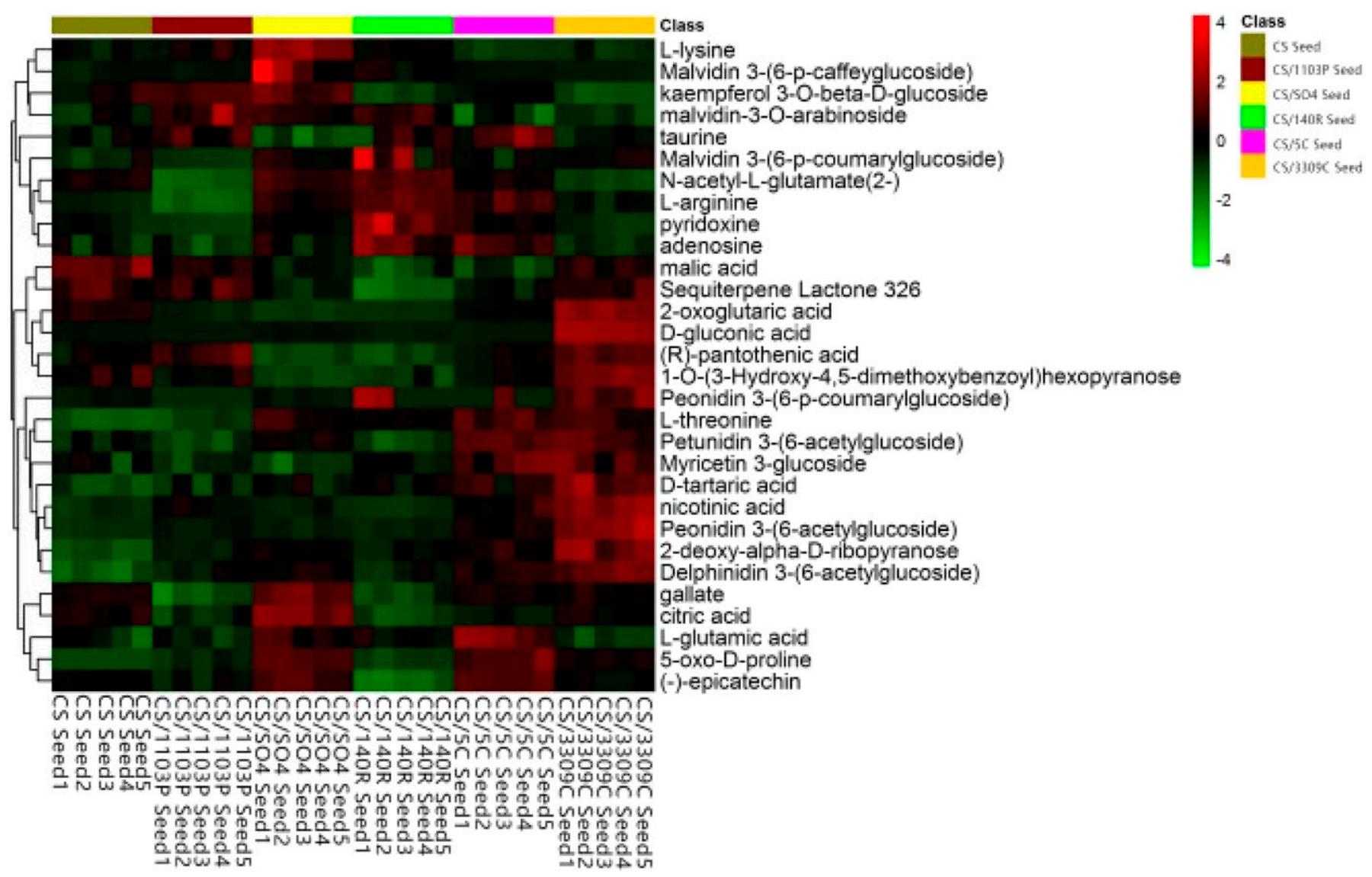

Fig. 5. Hierarchical clustering heatmap of the metabolites of 'Cabernet Sauvignon' (CS) grape seeds from various rootstock combinations.

which is similar to the findings of the present study. Therefore, it appears that rootstocks can significantly change the concentrations of organic acids in grape seeds of different cultivars grafted onto them.

Polyphenols affect various biological functions, including protection against predators, pathogens, and abiotic stress (Cheng et al., 2017). Strong antioxidant activity (e.g., because of picatechin and gallic acid esters) maintains normal physiological and biochemical processes in fruit and protects reactive oxygen species produced during abiotic stress (Koundouras et al., 2009). It also has been reported that ingesting foods that comprise polyphenolic antioxidants can provide anti-inflammatory effects, reduce the incidence of cardiovascular diseases, and exert anticancer effects (Morrison et al., 2014; Prince et al., 2016; Yoshioka et al., 2000). Anthocyanins, such as delphinidin glucoside and peony glucoside, affect the color and taste of wine (Vidal et al., 2004). It has been reported that anthocyanins in grape seeds also exert anticancer effects and improve cardiovascular and cerebrovascular functions (Pascual-Teresa and SanchezBallesta, 2008). Chemicals derived from grape seeds also provide active ingredients in medicines.

In the present study, it was demonstrated that all the grafting treatments had higher concentrations (in varying degrees) of delphinidin-3-(6-acetylglucoside) and paeonidin3-(6-p-coumarin glycoside) in the grape seeds than in the controls and that seeds from the $\mathrm{CS} /{ }^{\prime} \mathrm{SO} 4$ ' rootstock combination had significantly higher concentrations of epicatechin and gallate than the controls. Some studies have shown that the concentration of grape polyphenols is high in other cultivars of rootstocks (Cheng et al., 2017). Furthermore, the expression levels of genes responsible for the synthesis of VviPAL3-like, VviCHS3, VviLAR2, and VviUFGT anthocyanins are reported to be higher in grape seeds of the CS/' $\mathrm{M} 4$ ' rootstock combination than in those of the $\mathrm{CS} /{ }^{\prime} 1103 \mathrm{P}$ ' rootstock combination (Corso et al., 2016), which indicates that the synthesis of polyphenols in grape seeds can be improved by selecting appropriate rootstock combinations. However, the molecular mechanism of this synthesis needs further exploration.

It is well known that grapes are rich in vitamins. Reports on the effects of rootstocks on vitamin metabolism in grapes have mainly focused on vitamins $\mathrm{C}$ and $\mathrm{E}$. However, to the best of our knowledge, the effects of rootstocks on the vitamin B family have not been reported to date, despite the vitamin B family playing an important role in plant development. The human body must have, but cannot synthesize, several substances that prevent diseases (Chen and Xiong, 2005; Noctor, 2006; Said and Mohammed, 2006). The present study showed that the CS/' $1103 \mathrm{P}$ ' rootstock combination significantly increased the concentrations of nicotinic acid (vitamin B3 or vitamin PP) and pantothenic acid (vitamin B5), whereas the $\mathrm{CS} /{ }^{\prime} \mathrm{SO} 4$ ' rootstock combination significantly increased the concentration of pyridoxine (vitamin B6). These results suggest that the health benefits of grape seeds can be improved by using appropriate 
Table 2. Various metabolic pathways in 'Cabernet Sauvignon' grape seeds of various rootstock combinations.

\begin{tabular}{|c|c|c|c|c|c|c|c|}
\hline No. & Metabolic pathway & $\begin{array}{c}\text { Total } \\
\text { metabolites } \\
(\text { no. })^{\mathrm{z}}\end{array}$ & $\begin{array}{c}\text { Detected } \\
\text { metabolites } \\
(\text { no. })^{\mathrm{y}}\end{array}$ & $-\log (P)^{\mathrm{x}}$ & Impact $^{\mathrm{w}}$ & Pathway $^{\mathrm{v}}$ & Compounds $^{\mathrm{u}}$ \\
\hline$\overline{1}$ & Aminoacyl-transfer RNA biosynthesis & 67 & 4 & 4.0604 & 0 & ath00970 & $\mathrm{C} 00062, \mathrm{C} 00047, \mathrm{C} 00188, \mathrm{C} 00025$ \\
\hline 2 & Arginine and proline metabolism & 38 & 3 & 3.9579 & 0.27008 & ath00330 & $\mathrm{C} 00062, \mathrm{C} 00025, \mathrm{C} 00624$ \\
\hline 3 & Citrate cycle (TCA cycle) & 20 & 2 & 3.3016 & 0.16769 & ath00020 & $\mathrm{C} 00026, \mathrm{C} 00158$ \\
\hline 4 & $\begin{array}{l}\text { Alanine, aspartate and } \\
\text { glutamate metabolism }\end{array}$ & 22 & 2 & 3.1247 & 0.42528 & ath00250 & $\mathrm{C} 00026, \mathrm{C} 00025$ \\
\hline 5 & Taurine and hypotaurine metabolism & 5 & 1 & 2.5852 & 1 & ath00430 & $\mathrm{C} 00245$ \\
\hline 6 & Flavone and flavonol biosynthesis & 9 & 1 & 2.0267 & 0 & ath00944 & C12249 \\
\hline 7 & Lysine biosynthesis & 10 & 1 & 1.9287 & 0.07407 & ath00300 & $\mathrm{C} 00047$ \\
\hline 8 & Vitamin B6 metabolism & 11 & 1 & 1.8406 & 0 & ath00750 & $\mathrm{C} 00314$ \\
\hline 9 & beta-Alanine metabolism & 12 & 1 & 1.7609 & 0 & ath00410 & $\mathrm{C} 00864$ \\
\hline 10 & Nicotinate and nicotinamide metabolism & 12 & 1 & 1.7609 & 0 & ath00760 & $\mathrm{C} 00253$ \\
\hline 11 & Pantothenate and CoA biosynthesis & 14 & 1 & 1.6212 & 0.15 & ath00770 & C00864 \\
\hline 12 & Nitrogen metabolism & 15 & 1 & 1.5594 & 0 & ath00910 & $\mathrm{C} 00025$ \\
\hline 13 & Lysine degradation & 17 & 1 & 1.4487 & 0 & ath00310 & $\mathrm{C} 00047$ \\
\hline 14 & Glyoxylate and dicarboxylate metabolism & 17 & 1 & 1.4487 & 0.10544 & ath00630 & $\mathrm{C} 00158$ \\
\hline 15 & Pentose phosphate pathway & 18 & 1 & 1.3987 & 0 & ath00030 & $\mathrm{C} 00257$ \\
\hline 16 & Butanoate metabolism & 18 & 1 & 1.3987 & 0 & ath00650 & $\mathrm{C} 00025$ \\
\hline 17 & Valine, leucine and isoleucine biosynthesis & 26 & 1 & 1.0878 & 0 & ath00290 & $\mathrm{C} 00188$ \\
\hline 18 & Glutathione metabolism & 26 & 1 & 1.0878 & 0.07756 & ath00480 & $\mathrm{C} 00025$ \\
\hline 19 & Porphyrin and chlorophyll metabolism & 29 & 1 & 0.99963 & 0 & ath00860 & $\mathrm{C} 00025$ \\
\hline 20 & Glycine, serine and threonine metabolism & 30 & 1 & 0.97272 & 0.13697 & ath00260 & $\mathrm{C} 00188$ \\
\hline 21 & Flavonoid biosynthesis & 43 & 1 & 0.70212 & 0 & ath00941 & C09727 \\
\hline 22 & Purine metabolism & 61 & 1 & 0.47184 & 0.00256 & ath00230 & $\mathrm{C} 00212$ \\
\hline
\end{tabular}

${ }^{\mathrm{z}}$ Total number of metabolites in the target metabolic pathway.

${ }^{\mathrm{y}}$ Number of differential metabolites in the target metabolic pathway.

${ }^{\mathrm{x}}$ Significant difference $P$ value.

${ }^{\mathrm{w}}$ Metabolic pathway influence value.

${ }^{\mathrm{v}}$ Number of metabolic pathways in the Kyoto Encyclopedia of Genes and Genomes [KEGG (Kanehisa and Goto, 2000)].

${ }^{\mathrm{u}}$ Numbering of compounds in KEGG.



Fig. 6. Influence factor map of the metabolites of 'Cabernet Sauvignon' grape seeds from various rootstock combinations.

rootstocks. Furthermore, our study shows that various rootstock combinations can increase the concentrations of amino acids, organic acids, polyphenols, and vitamin B in grape seeds. Therefore, grape seed quality can be improved by selecting specific rootstock combinations for specific purposes, such as developing disparate attributes related to improvements in mouthfeel, nutritional properties, and even the efficacy of grape products.

\section{Conclusions}

This study used LC-MS and nontargeted metabolomic profiling to examine 30 metabolites produced in five grafted rootstock combinations and a self-rooted grafting CS grape control to conduct pathway enrichment analysis and screen for differences among treatments of metabolites in grape seeds. It found 22 significant metabolic pathways in the six rootstock combinations of CS grape seeds.

According to the nutrients contained in grape seeds, the SO4 rootstock in Xinjiang is a suitable rootstock for CS grape seeds. Various rootstock combinations change the nutrient composition of grape seeds by influencing several metabolic pathways: alanine-aspartic acid-glutamate metabolism, arginine-proline metabolism, and the TCA cycle.

Various rootstock combinations differ in the degree to which they increase the concentrations of amino acids, organic acids, polyphenols, and vitamin B in grape seeds. When CS is grafted onto the '1103P', '140R', 'SO4', '5C', and '3309C' rootstocks, the concentrations of delphinidin 3-(6-acetylglucoside), peonidin 3-(6-p-coumarylglucoside), L-threonine, and tartaric acid increased in the CS grape seeds, and subsequently, the quality, 
taste, nutritional concentration, and efficacy of these grape seeds may be improved.

The present study successfully identified characteristic markers for six types of rootstock combinations (all with CS as the scion) and revealed the influence of rootstocks on the metabolites in grape seeds, which provided a basis for determining the influence of various rootstock combinations. The additional breeding of grape rootstocks could provide important insights into improving the quality of derivative commercial products derived from grapes.

\section{Literature Cited}

Arapitsas, P., A. Della Corte, H. Gika, L. Narduzzi, F. Mattivi, and G. Theodoridis. 2016. Studying the effect of storage conditions on the metabolite content of red wine using HILIC LC-MS based metabolomics. Food Chem. 197(B):1331-1340.

Batista, L., S. Monteiro, V.B. Loureiro, A.R. Teixeira, and R.B. Ferreira. 2010. Protein haze formation in wines revisited. The stabilising effect of organic acids. Food Chem. 122:1067-1075.

Boulesteix, A.L. and K. Strimmer. 2007. Partial least squares: A versatile tool for the analysis of high-dimensional genomic data. Brief. Bioinform. 8:32-44.

Bueno, M.J.M., F.J. Diaz-Galiano, L. Rajski, V. Cutillas, and A.R. Fernandez-Alba. 2018. A non-targeted metabolomic approach to identify food markers to support discrimination between organic and conventional tomato crops. J. Chromatography 1546:66-76.

Chen, H. and L. Xiong. 2005. Pyridoxine is required for post-embryonic root development and tolerance to osmotic and oxidative stresses. Plant J. 44:396-408.

Cheng, J., L. Wei, J. Mei, and J. Wu. 2017. Effect of rootstock on phenolic compounds and antioxidant properties in berries of grape (Vitis vinifera L.) cv. 'Red Alexandria'. Scientia Hort. 217:137-144.

Consonni, R., L.R. Cagliani, M. Stocchero, and S. Porretta. 2009. Triple concentrated tomato paste: Discrimination between Italian and Chinese products. J. Agr. Food Chem. 57:4506-4513.

Corso, M., A. Vannozzi, F. Ziliotto, M. Zouine, E. Maza, T. Nicolato, N. Vitulo, F. Meggio, G. Valle, M. Bouzayen, M. Muller, S. MunneBosch, M. Lucchin, and C. Bonghi. 2016. Grapevine rootstocks differentially affect the rate of ripening and modulate auxin-related genes in cabernet sauvignon berries. Front. Plant. Sci. 7:69.

De Vos, R.C.H., S. Moco, A. Lommen, J.J.B. Keurentjes, R.J. Bino, and R.D. Hall. 2007. Untargeted large-scale plant metabolomics using liquid chromatography coupled to mass spectrometry. Nat. Protoc. 2:778-791.

Gordana, Z., G. Dejan, Š. Katarina, K. Dragana, and M. Nebojša. 2019. Grape seed polyphenols and fatty acids of autochthonous prokupac vine variety from Serbia. Chem. Biodivers. 16:e1900053.

Gromski, P.S., H. Muhamadali, D.I. Ellis, Y. Xu, E. Correa, M.L. Turner, and R. Goodacre. 2015. A tutorial review: Metabolomics and partial least squares-discriminant analysis-a marriage of convenience or a shotgun wedding. Anal. Chim. Acta 879:10-23.

Harb, J., S. Alseekh, T. Tohge, and A.R. Fernie. 2015. Profiling of primary metabolites and flavonols in leaves of two table grape varieties collected from semiarid and temperate regions. Phytochemistry $117: 444-455$.

Hela, H., B.R. Iness, O. Ines, A.W. Wissem, Z. Hassen, N. Zoghlami, N.A. Khan, R. Ksouri, and M.S. Tounsi. 2018. Biochemical characterization and antioxidant activity of grape (Vitis vinifera L.) seed oils from nine Tunisian varieties. J. Food Biochem. 42:e12595.

International Organisation of Vine and Wine. 2018. Global state of conditions report: Developments and trends. 24 Apr. 2018. $<\mathrm{http}: / /$ www.oiv.int/public/medias/5948/en-oiv-press-release-24-april2018.pdf>.

Ji, N., D. Min, F. Li, S. Shao, and X. Zhang. 2018. Role of nitric oxide synthase pathway in arginine-induced disease resistance in postharvest tomato fruit. Shipin Kexue 39:250-257.
Jin, Z.X., H. Sun, T.Y. Sun, Q.J. Wang, and Y.X. Yao. 2016 a. Modifications of 'Gold Finger' grape berry quality as affected by the different rootstocks. J. Agr. Food Chem. 64:4189-4197.

Jin, Z.X., T.Y. Sun, H. Sun, Q.Y. Yue, and Y.X. Yao. 2016 b. Modifications of 'Summer Black' grape berry quality as affected by the different rootstocks. Scientia Hort. 210:130-137.

Jogaiah, S., D.P. Oulkar, K. Banerjee, J. Sharma, A.G. Patil, S.R. Maske, and R.G. Somkuwar. 2013. Biochemically induced variations during some phenological stages in thompson seedless grapevines grafted on different rootstocks. S. Afr. J. Enol. Vitic. 34:36-45. Joshua, V., F. Alessandro, P. Enrico, S. Paolo, G.M. Ilce, F. Rachele, R. Laura, and S. Paolo. 2020. Increase in seed tannin extractability and oxidation using a freeze-thaw treatment in cool-climate grown red (Vitis vinifera L.) cultivars. Food Chem. 308:125571.

Kanehisa, M. and S. Goto. 2000. KEGG: Kyoto encyclopedia of genes and genomes. Nucleic Acids Res. 28:27-30.

Koundouras, S., E. Hatzidimitriou, M. Karamolegkou, E. Dimopoulou, S. Kallithraka, J.T. Tsialtas, E. Zioziou, N. Nikolaou, and Y. Kotseridis. 2009. Irrigation and rootstock effects on the phenolic concentration and aroma potential of Vitis vinifera L. cv. Cabernet Sauvignon grapes. J. Agr. Food Chem. 57:7805-7813.

Lee, J.E., B.J. Lee, J.O. Chung, J.A. Hwang, S.J. Lee, C.H. Lee, and Y.S. Hong. 2010. Geographical and climatic dependencies of green tea (Camellia sinensis) metabolites: A (1)H NMR-based metabolomics study. J. Agr. Food Chem. 58:10582-10589.

Leng, X.P., H.F. Jia, X. Sun, L.F. Shangguan, Q. Mu, B.J. Wang, and J.G. Fang. 2015. Comparative transcriptome analysis of grapevine in response to copper stress. Sci. Rep. (UK) 5:17749.

Morrison, M., R. van der Heijden, P. Heeringa, E. Kaijzel, L. Verschuren, R. Blomhoff, T. Kooistra, and R. Kleemann. 2014. Epicatechin attenuates atherosclerosis and exerts anti-inflammatory effects on diet induced human-crp and nfkb in vivo. Atherosclerosis 233:149-156.

Narduzzi, L., J. Stanstrup, and F. Mattivi. 2015. Comparing wild American grapes with Vitis vinifera: A metabolomics study of grape composition. J. Agr. Food Chem. 63:6823-6834.

Noctor, G. 2006. Metabolic signalling in defence and stress: The central roles of soluble redox couples. Plant Cell Environ. 29:409-425.

Pascual-Teresa, S.D. and M.T. Sanchez-Ballesta. 2008. Anthocyanins: From plant to health. Phytochem. Rev. 7:281-299.

Prince, P.D., C.R. Lanzi, J.E. Toblli, R. Elesgaray, P.I. Oteiza, C.G. Fraga, and M. Galleano. 2016. Dietary (-)-epicatechin mitigates oxidative stress, NO metabolism alterations, and inflammation in renal cortex from fructose-fed rats. Free Radic. Biol. Med. 90:35-46.

Pulko, B., S. Vrsic, and J. Valdhuber. 2012. Influence of various rootstocks on the yield and grape composition of Sauvignon Blanc. Czech J. Food Sci. 30:467-473.

Qi, X., X. Chen, and Y. Wang. 2011. Plant metabolomics: Methods and applications. 1st ed. Chem. Ind. Press, Beijing, China.

Rieping, M. and T. Hermann. 2006. L-Threonine, p. 71-92. In: V.F. Wendisch (ed.). Amino acid biosynthesis-Pathways, regulation and metabolic engineering. Microbiol. Monogr. Vol. 5. Springer, Berlin, Germany.

Said, H.M. and Z.M. Mohammed. 2006. Intestinal absorption of watersoluble vitamins: An update. Curr. Opin. Gastroenterol. 22:140-146.

Sangster, T., H. Major, R. Plumb, A.J. Wilson, and I.D. Wilson. 2006. A pragmatic and readily implemented quality control strategy for HPLC-MS and GC-MS-based metabonomic analysis. Analyst 131:1075-1078.

Son, H.S., K.M. Kim, F. van den Berg, G.S. Hwang, W.M. Park, C.H. Lee, and Y.S. Hong. 2008. 1H nuclear magnetic resonance-based metabolomic characterization of wines by grape varieties and production areas. J. Agr. Food Chem. 56:8007-8016.

Suriano, S., V. Alba, D. Di Gennaro, M.S. Suriano, M. Savino, and L. Tarricone. 2016. Genotype/rootstocks effect on the expression of anthocyanins and flavans in grapes and wines of Greco Nero n. (Vitis vinifera L.). Scientia Hort. 209:309-315. 
Tarachiwin, L., A. Katoh, K. Ute, and E. Fukusaki. 2008. Quality evaluation of Angelica acutiloba Kitagawa roots by $1 \mathrm{H}$ NMR-based metabolic fingerprinting. J. Pharmaceut. Biomed. 48:42-48.

Thévenot, E.A., A. Roux, Y. Xu, E. Ezan, and C. Junot. 2015. Analysis of the human adult urinary metabolome variations with age, body mass index, and gender by implementing a comprehensive workflow for univariate and OPLS statistical analyses. J. Proteome Res. 14:3322-3335.

Trad, M., C. Le Bourvellec, H.B. Hamda, C.M.G.C. Renard, and M. Harbi. 2017. Flavan-3-ols and procyanidins in grape seeds: Biodiversity and relationships among wild and cultivated vines. Euphytica 213:242.

Vidal, S., L. Francis, A. Noble, M. Kwiatkowski, V. Cheynier, and E. Waters. 2004. Taste and mouth-feel properties of different types of tannin-like polyphenolic compounds and anthocyanins in wine. Anal. Chim. Acta 513:57-65.
Want, E.J., I.D. Wilson, H. Gika, G. Theodoridis, R.S. Plumb, J. Shockcor, E. Holmes, and J.K. Nicholson. 2010. Global metabolic profiling procedures for urine using UPLC-MS. Nat. Protoc. 5:1005-1018.

Yoshioka, K., T. Kata Oka, T. Hayashi, M. Hasegawa, Y. Ishi, and H. Hibasami. 2000. Induction of apoptosis by gallic acid in human stomach cancer KATOIII and colon adenocarinoma COLO205 cell lines. Oncol. Rep. 7:1221-1224.

Zerbib, M., G. Cazals, C. Enjalbal, and C. Saucier. 2018. Identification and quantification of flavanol glycosides in Vitis vinifera grape seeds and skins during ripening. Molecules 23:2745.

Zhao, H.X., P. Xie, X.L. Li, W.Q. Zhu, X.D. Sun, X.R. Sun, X.T. Chen, L.G. Xing, and J.M. Yu. 2015. A prospective phase II trial of EGCG in treatment of acute radiation-induced esophagitis for stage III lung cancer. Radiother. Oncol. 114:351-356. 